Check for updates

Cite this: RSC Adv., 2017, 7, 22927

Received 6th February 2017

Accepted 8th April 2017

DOI: 10.1039/c7ra01520h

rsc.li/rsc-advances

\section{Teratogenic, cardiotoxic and hepatotoxic properties of related ionic liquids reveal the biological importance of anionic components $\uparrow$}

\author{
Aditi Pandey, (D) Mary Krishna Ekka, (D) Shashi Ranjan, (D) Souvik Maiti (D) \\ and Chetana Sachidanandan (iD *
}

\begin{abstract}
Ionic liquids are fast becoming the solvent of choice for a number of diverse chemical, industrial and biological processes. Although they are considered a 'greener' alternative to conventional solvents, their effects on biological systems remain poorly understood. The zebrafish, a small fresh-water fish, is an ideal model for testing the effects of small molecules on whole organism assays. Because of conservation of molecular pathways between human and zebrafish, studies on zebrafish can lead to direct insights into human biology. We have evaluated the toxic effects of five different but related ionic liquids on zebrafish embryonic assays. We have discovered two related ionic liquids that show strong cardiotoxic and hepatotoxic effects suggesting previously unanticipated effects on health. Comparisons of ionic liquids with different cationic but the same anionic components suggest that both the anionic and the cationic components play important roles in determining the biological effects of an ionic liquid.
\end{abstract}

\section{Introduction}

Ionic liquids (ILs) are salts where the ions are poorly coordinated resulting in these salts remaining in a liquid form below $100{ }^{\circ} \mathrm{C}$, or even at room temperature. ${ }^{1}$ Their unique properties have made them an ideal replacement for conventional solvents in electrochemistry, ${ }^{2}$ organic and inorganic transformations, ${ }^{3,4}$ biotechnology processes ${ }^{3,5,6}$ and bio-transformations ${ }^{5}$ and as solar cell electrolytes, ${ }^{7}$ and supported phases in organic chemistry. ${ }^{8}$ The new generation ionic liquids are increasingly being explored for their biological applications.

Studies on the biological activity of ILs and their applications in pharmaceutics and medicine have become a major area of research. ${ }^{9}$ Initial toxicity experiments on microorganisms revealed that ionic liquids may have great potential as antimicrobials. ${ }^{\mathbf{1 0 , 1 1}}$ This is especially valuable in the current climate where microbes are evolving resistance to most traditional classes of anti-microbial drugs. Ionic liquid cytotoxicity has also been observed on numerous cancer cell lines suggesting their utility as anti-cancer drugs. ${ }^{12-14}$ The unique properties of ionic liquids make them excellent candidates for exploring potential uses such as for drug delivery, increasing solubility and bioavailability of drugs and as drug candidates themselves. ${ }^{9}$ Amidst the promise of new beneficial biological activities that relies on their interaction with the human body, it is also

CSIR-Institute of Genomics and Integrative Biology, South Campus, New Delhi 110025, India.E-mail: chetana@igib.res.in; chetana@igib.in

$\dagger$ Electronic supplementary information (ESI) available. See DOI: 10.1039/c7ra01520h essential to assess the toxicity and safety of these molecules. With such proposed widespread usage, it is also inevitable for ILs to be released into environment. A thorough assessment of their effects on health and environment is urgently needed. In terms of their ecological impact, several studies are already available that touch upon the toxicity of ILs on unicellular organisms and invertebrates. Majority of these are toxicological assessment of various ILs on the aquatic ecosystem; assessing the impact on organisms such as Vibrio fischeri, ${ }^{\mathbf{1 0}, 15-17}$ Daphnia magna $^{15,16,18}$ and Selenastrum capricornutum..$^{18,19}$

Physiochemical properties of ILs such as solubility, stability and their interaction with surrounding media have a determining effect on their biological activity. According to a 2014 study using bis(trifluoromethylsulfonyl)amide anion ([NTf2 $]^{-}$) and varying cations, showed that the water solubility of ILs decreased in the order imidazolium ILs $>$ pyrrolidinium ILs $>$ pyridinium ILs $>$ piperidinium ILs. ${ }^{20}$ While another study with bromide-based anions combined with various cations found that the toxicity on Vibrio fischeri and Daphnia magna decreased in the order pyridinium $\sim$ imidazolium $>$ piperidinium $>$ pyrrolidinium. ${ }^{21}$ Thus, illustrating that the relationship is not based on one property of the IL but rather is more complex. Various systems have been used to evaluate the role of the chain length in the biological toxicity. Earlier studies in rat cells lines, ${ }^{22}$ luminescent bacteria ${ }^{10}$ and Vibrio fischeri ${ }^{23}$ found that as the alkyl chain length increased, the lethal effects of the ionic liquids increased. This is also supported in other studies where it was found that longer alkyl chains had increased toxicity in human lung carcinoma A549 cell line. ${ }^{14}$ Results however, are ambiguous over the contribution of the cationic and anionic 
group of the $\mathrm{IL}^{24}$ In fact, studies with over 200 ILs have concluded that cationic components are the major contributors towards toxicity while anions play only a secondary role. ${ }^{24}$ Other studies have concluded that features like long hydrocarbon side chain, combined with bulky hydrophobic headgroup, or a bulky anion, all contribute to increasing degrees of toxicity. ${ }^{25-27}$ Quantitative structure-activity relationship (QSAR) studies have been performed to delineate the involvement of the structural features of the cationic/anionic head groups in the cytotoxicity using the ionic liquids' empirical formulas and molecular weights as descriptors. ${ }^{28,29}$ Similar quantitative structuretoxicity relationship (QSTR) studies indicated that the increase in the heavy atom count of the anionic group leads to an increase in the toxicity. ${ }^{24}$ Since NTf2 is comparatively a large anion, its charge density is more localized than smaller anions like tetrafluoroborate. Large molecules like this replace more water and are highly hydrophobic, hence contributing to higher toxicity. This is well supported in experiments done on human lung carcinoma A549 cell line with various the anionic group bis(trifluoromethyl sulfonyl)imide exhibiting one of the lowest EC50 values. ${ }^{14}$ NTf2 salts were also found to be more toxic than halide salts in cytotoxic studies done in HeLa cell line. ${ }^{\mathbf{1 4 , 3 0}} \mathrm{A}$ similar effect was also observed in case of bacterial toxicity studies where Ntf2 containing ILs were generally more toxic to microorganisms as compared to other anions. ${ }^{31}$ In this case, both cationic and anionic moiety can insert themselves into the lipid bilayer of the cell membrane, thus perturbing the membrane structure to cause swelling of the bilayer and alteration of its permeability properties, causing IL toxicity. ${ }^{\mathbf{1 4 , 2 7}}$ There are two possible mechanisms of cytotoxicity induced by the anionic component of the IL. First is the lipophilicity of the anionic moiety; the other is the intrinsic ability of the anion to form hydrolysis products. ${ }^{24}$ This especially holds true for those anions possessing a fluoride moiety. ${ }^{22,30}$

Most of the studies discussed thus far are on microorganisms and cultured cells and these impart only limited utility in understanding the toxic effects of ionic liquids on human health. Egorova ${ }^{9}$ and Frade, ${ }^{32}$ in their respective reviews on the biological activity of ionic liquids have independently remarked upon the complex and dramatically different susceptibilities of different biological systems to ILs. Reiterating that, toxicity depends on the studied model and to assess toxicity of any IL, studies on different models and environmental conditions are needed. To model health hazards of ionic liquids on humans, model organisms such as mice and rat have been very useful. Acute and subchronic toxicity of the IL didecyldimethylammonium saccharinate has been determined in rats. ${ }^{33}$ Other acute toxicity studies with tetrabutylammonium chloride IL on mice have found severe histological damage of liver and kidney upon treatment. ${ }^{34}$ However, other studies have also found that 3-hexyloxymethyl-1-methylimidazolium tetrafluoroborate was not toxic to Wistar rats at the concentrations tested. ${ }^{35}$ Bailey et al. evaluated the effects of prenatal exposure of mice to 1-butyl-3-methylimidazolium chloride. When mothers were exposed to the IL, the fetal weight was found to reduce. At the highest dosage, malformations were also observed indicating teratogenicity. ${ }^{36} \mathrm{Li}$ et al. have studied the effect of 1-methyl-3-octylimidazolium bromide on the early embryonic development of the frog Rana nigromaculata and found strong toxic effects on embryogenesis stages. ${ }^{37}$ These studies have made a beginning to testing ILs on vertebrate model organisms that can model human biology.

In the last many years zebrafish (Danio rerio) has emerged as an ideal model organism to design high-throughput chemical screens with relevance to human embryonic developmental biology. ${ }^{38}$ The genome and basic physiology of this small fresh water fish is highly conserved with that of humans. ${ }^{39,40}$ Zebrafish has been successfully used to detect and dissect the toxicity of environmental toxins and pollutants. ${ }^{41}$ The whole organism assays in zebrafish embryos not only allows us to assess toxicity of compounds and determine their lethal dose but also decipher the effect of sub-lethal concentrations on various organ systems and on embryonic development. Also, fish being important as human food and their distribution in the aquatic environment; exploring the effects of ILs on fish could help evaluate the toxic effects on the food chain.

Only a limited number of studies on the bioactivity and toxicity of ILs have used zebrafish as a model. ${ }^{42-45}$ Pretti et al. ${ }^{42}$ in 2006 examined acute toxicity of ionic liquids in adult zebrafish, which revealed that ionic liquids cause completely different effects on fish compared to other model organisms studied till date. They found that imidazolium, pyridinium and pyrrolidinium showed higher LC50 values (>100 $\mathrm{mg} \mathrm{l}^{-1}$ ) whereas ammonium salts showed remarkably lower LC50 values. Moreover, their histological evaluation of adult zebrafish showed skin alterations such as epithelial hyperplasia with single keratinocyte vesiculation and wide erosions together with dis-epithelialization of gill lamellae. Another recent study has examined the toxicity of select imidazolium ILs in other marine and fresh water fish and found them to be nontoxic ${ }^{\mathbf{4 6}}$ while a study by $\mathrm{Du}$ et al. $^{\mathbf{4 4}}$ found that 1-octyl-3-methylimidazolium bromide can induce oxidative stress and DNA damage in adult zebrafish.

In this study we have utilized the transparent nature of zebrafish embryos and the powerful genetic tools available, to study the effect of five different but related ILs on zebrafish. We have used the zebrafish system to determine the toxicity and biological effects of different ILs on embryonic development. We have examined the effect of short and long exposure to ILs on transgenic zebrafish and have discovered strong deleterious effects on heart function and liver health of two ILs that share the same anion. Our data suggests that anionic components of ILs also may be important contributors to organ toxicity of ILs during development and function.

\section{Methods and materials}

All ionic liquids were obtained from Sigma-Aldrich, India. Here we use five ILs: 1-butyl-3-methylimidazolium tetrafluoroborate (IL1), 1-butyl-3-methylimidazolium hexafluorophosphate (IL2), 1butyl-1-methylpyrrolidinium bis(trifluoromethylsulfonyl)imide (IL3), 1-ethyl-3-methylimidazolium bis(trifluoromethylsulfonyl) imide (IL4) and 1-butyl-4-methylpyridinium tetrafluoroborate (IL5). 


\section{Cell viability assay}

Cell viability was measured using an MTT assay $^{\mathbf{4 7}}$ and performed on human breast adenocarcinoma cell line MCF-7. Stock solutions of ionic liquids were prepared in the culture medium according to their solubility. A stock solution of 3(4,5-dimethylthiazol-2-yl)-2,5-diphenyltetrazolium bromide salt (MTT, Fluka) was made at $5 \mathrm{mg} \mathrm{ml}^{-1}$ in a phosphatebuffered saline solution (PBS, $1 \times, \mathrm{pH} 7.4$ ). The working solution for MTT was $0.5 \mathrm{mg} \mathrm{m}{ }^{-1}$. Cells were cultured in $25 \mathrm{~cm}^{2}$ flasks (Corning) in high glucose DMEM (Dulbecco's Modified Eagle's Medium), supplemented with $10 \%$ fetal bovine serum (FBS) at $37{ }^{\circ} \mathrm{C}$ in a humidified chamber at $5 \% \mathrm{CO}_{2}$. Upon reaching confluence, cells were seeded at a final concentration of $1 \times 10^{5}$ cells into 96-well plates (Nunc) with blanks, controls and IL dilutions with three replicates each. Total volume in each well was $200 \mu \mathrm{l}$. The cells were treated for 24 hours with the desired IL concentration gradient. After incubation, the culture media was substituted by fresh media containing MTT solution (working $0.5 \mathrm{mg} \mathrm{ml}^{-1}$ ) and incubated for a further 4 hours at $37{ }^{\circ} \mathrm{C}$. After the formation of formazan crystals, this media was discarded and the crystals dissolved in $200 \mu \mathrm{l}$ of dimethylsulfoxide. Subsequently, the optical density was measured by using a microplate reader (Tecan Infinite M200 Pro Multi-plate Reader) at $570 \mathrm{~nm}$ to detect the concentration of formazan present. Cell viability was calculated as the percentage of viable cells, which were treated with ILs versus untreated cells. Origin software was used for data analysis and plotting the graphs.

\section{Zebrafish lines and maintenance}

Zebrafish (Danio rerio) were raised, bred and maintained at $28.5{ }^{\circ} \mathrm{C}$ under standard conditions as described. ${ }^{48}$ Embryos were staged by morphological features using criteria previously described. ${ }^{49}$ For fluorescence imaging, embryos were raised in 1-phenyl-2-thiourea (PTU) $(0.003 \%$ in embryo medium) to inhibit pigment formation. All zebrafish handling and experiments were performed in accordance to protocols approved for BSC0124 by the Institutional Animal Ethics Committee (IAEC) of the CSIR-Institute of Genomics and Integrative Biology, India under the rules and regulations set by the Committee for the Purpose of Control and Supervision of Experiments on Animals (CPCSEA), Ministry of Environment, Forests and Climate Change, Government of India.

The zebrafish lines used in this study are wildtype (TubingenTU) and $T g$ (fabp10a:GAL4-VP16, myl7:cerulean)::(UAS:nfsBCherry). The fabp10a:GAL4-VP16, myl7:cerulean construct was made from the pCH Gtwy G4VP16 plasmid (a kind gift from Michael Nonet http://www.dbbs.wustl.edu/RIB/NonetMicL). 2816 bp of the $5^{\prime}$-flanking region (chr16:52623514-52626329) of the fabp10a gene was PCR amplified and cloned into the $\mathrm{pCH}$ Gtwy G4VP16 plasmid by gateway cloning (Invitrogen). Tg(fabp10a:GAL4-VP16, myl7:cerulean) line was produced by microinjection of this construct into one-cell stage zebrafish embryos. This line was crossed with $T g\left(U A S: n f s B\right.$-Cherry), ${ }^{50}$ to generate the double transgenic line $T g$ (fabp10a:GAL4-VP16, myl7:cerulean)::(UAS:nfsB-Cherry).

\section{Chemical treatment of zebrafish embryos}

Wildtype zebrafish embryos were exposed to different dilutions of the ILs in water from 6 hours onwards (day 0) to day 4 for both toxicity profile and the teratogenicity assay. The different concentrations of each ionic liquid used were $0.125 \mathrm{mM}, 1.25 \mathrm{mM}$, $2.5 \mathrm{mM}, 5 \mathrm{mM}, 10 \mathrm{mM}$ and $20 \mathrm{mM}$ and were compared with water. For cardio-toxicity assays, the Tg(fabp10a:GALA-VP16, myl7:cerulean)::(UAS:nfsB-Cherry) embryos were treated at day 1 and heart rate was measured on day 2 and also treated at 2.5 day for 16 hours and heart rate measured.

For hepatotoxicity assays, the $T g$ (fabp10a:GAL4-VP16, myl7:cerulean)::(UAS:nfsB-Cherry) embryos were treated on day 3 for 16 hours. The treatment of older embryos i.e. more than day 1 was done specifically for IL3 and IL4 to visualize the effects on organs. The concentration used at these stages was $2.5 \mathrm{mM}$ unless otherwise mentioned. In each experiment, each condition or concentration the number $(N)$ of embryos used was $>50$. Each experiment was repeated on three or more independent occasions. Average from $>=3$ experiments were calculated for analysis.

Observation and imaging of phenotypes were done using Zeiss Stemi 2000-C stereomicroscope with AxiocamICc1 and Zeiss AxioScope A1 fluorescence microscope with Axiocam HRc. All figures were prepared using Adobe Photoshop®.

MS Excel was used for data analysis and plotting survival graphs of zebrafish embryos. For heart rate measurement, graph pad prism was used for the analysis. For statistical significance of data, $p$-values were calculated by preforming unpaired student $t$-test for the data.

\section{Quantitative real-time PCR}

Total RNA was extracted by homogenizing 20 pooled embryos using Trizol reagent (Invitrogen). Quantitative real time PCR was carried out as described on the Roche LightCycler VR RealTime PCR System. ${ }^{51}$ Statistical analyses on normalized data were performed using 2-DDCT algorithm. ${ }^{51,52}$ All genes were normalized against $R P L 13 \alpha$. Graph pad prism was used for the analysis. For statistical significance of data, $p$-values were calculated by preforming unpaired student $t$-test for the data. All primers used in this study are listed in Table S6. $\dagger$

\section{Alcian blue staining}

Alcian blue stain was used to visualize the structure of the craniofacial skeleton of zebrafish as described previously. ${ }^{53}$

\section{Results and discussion}

Here we consider five ILs (Fig. 1): 1-butyl-3-methylimidazolium tetrafluoroborate (IL1), 1-butyl-3-methylimidazolium hexafluorophosphate (IL2), 1-butyl-1-methylpyrrolidinium bis(trifluoromethylsulfonyl)imide (IL3) and 1-ethyl-3-methylimidazolium bis(trifluoromethylsulfonyl)imide (IL4) and 1-butyl-4methylpyridinium tetrafluoroborate (IL5) (Fig. 1). IL1 and IL2 have the same cationic group i.e., 1-butyl-3-methylimidazolium with different anionic groups head groups, i.e. hexafluorophosphate and tetrafluoroborate respectively. On the other 


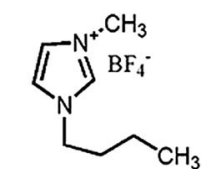

1-butyl-3-methylimidazolium tetrafluoroborate (IL1)

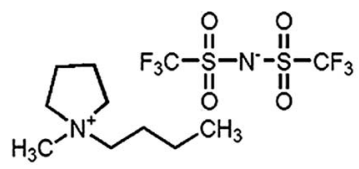

1-butyl-1-methylpyrrolidinium bis(trifluoromethylsulfonyl)imide (IL3)

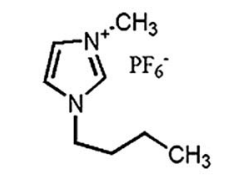

1-butyl-3-methylimidazolium exafluorophosphate (IL2)

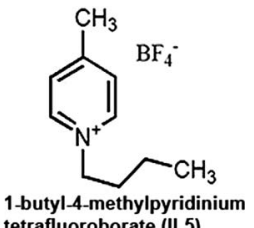

tetrafluoroborate (IL5)

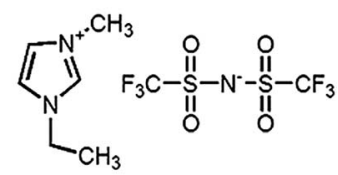

Fig. 1 Structure of ionic liquids tested in this study. IL1 and IL2 have same cationic group i.e., 1-butyl-3-methylimidazolium with different anionic groups head groups, i.e. hexafluorophosphate and tetrafluoroborate respectively. On the other hand, IL3 and IL4 have same anionic group (bis(trifluoromethylsulfonyl)imide) with different cationic groups, i.e. 1-butyl-1-methylpyrrolidinium and 1-ethyl-3-methylimidazolium respectively. Similarly IL1 and IL5 have the same anionic group (tetrafluoroborate) with different cationic groups, i.e. 1-butyl-3methylimidazolium and 1-butyl-4-methylpyridinium respectively.

hand, IL3 and IL4 have same anionic group (bis(trifluoromethylsulfonyl)imide) with different cationic groups, i.e. 1butyl-1-methylpyrrolidinium and 1-ethyl-3-methylimidazolium respectively. Similarly IL1 and IL5 have the same anionic group (tetrafluoroborate) with different cationic groups.

These ILs have been selected for their potential for use in biological and chemical processes. IL1 and IL2 has been shown be useful to improve the synthesis of naproxen and pravadoline, non-steroidal anti-inflammatory agents, respectively. ${ }^{54}$ IL2 has been demonstrated to be useful for DNA quantification. ${ }^{55}$ In fact, in the last many years, number of reports have emerged showcasing applications of IL1 and IL2 in various biological processes as solubility enhancers, emulsifiers, co-solvents, reaction media and catalysts. ${ }^{9}$ The anions used in this study were also selected based on their utility in a number of different applications such as for enhancing solubility, stabilizing drugs and proteins, for catalysis, for enantioselectivity and drug delivery systems. ${ }^{9}$

MTT assay was performed using MCF-7, to examine the cytotoxicity of ILs. Out of the ILs tested, IL2 was the most toxic (Fig. S1a and $\mathrm{b}^{\dagger}$ ) with approximately $98 \%$ cell death at $20 \mathrm{mM}$ concentration. This IL was the most potent with an EC50 value of $1.8 \mathrm{mM}$ (Fig. S1a $\dagger$ ). IL3 and IL4 were also very toxic (EC50 $3.5 \mathrm{mM}$ and $3.2 \mathrm{mM}$ respectively) which could be because of the shared bis (trifluoromethylsulfonyl)imide group as the anion (Fig. S1a and $\mathrm{b} \dagger$ ). IL1 and IL5 exhibited similar toxicity (EC50 $\sim 4.4 \mathrm{mM}$ and $4.8 \mathrm{mM}$ respectively) (Fig. S1a and $\mathrm{b}^{\dagger}$ ). These two ILs share the same anionic counterpart, tetrafluoroborate while the cationic component differs with IL1 having an imidazolium core and IL5 having a pyridinium core. Toxicity of ILs has been examined in other cell lines also. We compared the cytotoxicity of ILs on HeLa and rat leukemia cell line IPC-81 with our results on MCF-7. In these studies, ILs containing the bis(trifluoromethylsulfonyl) imide group were found to be more toxic in HeLa cells ${ }^{30}$ compared to other anions $(\mathrm{EC} 50 \sim 1.8 \mathrm{mM}, 48 \mathrm{~h}$ ) while IPC-81 showed similar toxicity profiles with MCF-7. ${ }^{24}$ ILs having tetrafluoroborate as anion also showed toxicity similar to our experiment. In one report the hexafluorophosphate containing IL showed moderate toxicity $(\mathrm{EC} 50=13.9 \mathrm{mM})$ towards HeLa cells while we got contrasting results with maximum toxicity reported by IL-2 in MCF-7 cells. ${ }^{30}$ However, EC50 values were found to vary with time, with different values reported after $24 \mathrm{~h}$ and $48 \mathrm{~h}$ of treatment. ${ }^{30}$

Since, the cellular toxicity can be very different from toxicity and lethality at the organism level, we used zebrafish whole organism assays. Zebrafish embryos were incubated in varying concentrations of ILs from 6 hours after fertilization (day 0) till day 4. During this period their viability was monitored at regular intervals. Percentage survival of IL1 treated embryos showed a concentration and time dependent response (Fig. S1c and Table $\mathrm{S} 1 \dagger)$. At $20 \mathrm{mM}$, the highest concentration tested, all embryos were dead on day 1 , indicating acute toxicity. However, with decreasing concentrations tested, the survival improved viz. $14 \%, 22 \%$ and $45 \%$ percent survival with $10 \mathrm{mM}, 5 \mathrm{mM}$ and $2.5 \mathrm{mM}$ respectively. IL1 has been previously shown to be toxic to wheat seedlings ${ }^{56}$ (at the concentration of $0.9 \mathrm{mM}$ or more, 24 h). IL2 was even more toxic with a $15 \%$ survival at $1.25 \mathrm{mM}$ (Fig. S1d and Table S2 $\dagger$ ). In contrast, IL3 and IL4 elicited effects due to prolonged exposure, on the survival of embryos (Fig. S1e and $\mathrm{f}$, Table S3 and $\mathrm{S} 4 \dagger$ ). On day 1, the survival was approximately $74 \%$ at $20 \mathrm{mM}$, while it dropped dramatically with time. IL5 had an intermediate effect, being more acute than IL3 and IL4, but less than IL1 and IL2 (Fig. S1g and Table S5†).

Thus, out of the five ILs tested in this study, IL1 and IL2 appear to have the most detrimental effects on embryos. This similarity could be attributed to their shared imidazolium based cationic core. ILs containing imidazolium have previously shown to be more toxic than other aromatic head groups such as pyridinium ${ }^{\mathbf{1 5}}$ (present in IL5) and non-aromatic head groups such as pyrrolidinium, ${ }^{57}$ as in IL3. The alkyl chain length of IL1 and IL2 was also longer than that of IL4. However, IL2 displayed the most potent effects on the biological systems tested here. This result suggests that the anion in IL2 may be responsible for the increased toxicity of IL2. IL1 and IL5 share a common anion, tetrafluoroborate. Salts containing TFB show one of the most potent toxic effects on cells. $^{58}$ Their difference in biological effect indicates that the toxicity in IL1 is most likely due to the cationic component. Also, due to higher symmetry of the pyridinium cation, IL5 could be less toxic than ILs containing an imidazolium cation. ${ }^{24}$ After IL2, ILs containing bis(trifluoromethylsulfonyl)imide group as the anion i.e. IL3 and IL4 were the most toxic to MCF-7 cells. However, IL3 and IL4 exhibited effects due to prolonged exposure, on the survival of zebrafish embryos and least acute toxicity. Thus, from comparison our studies on cells and the zebrafish we conclude that caution should be exercised in extrapolating toxicity data from cells to organisms, because the correlation is not straight forward.

The whole organism screen in zebrafish embryos not only allows us to assess toxicity of compounds and determine their lethal dose but also decipher effect of sub-lethal concentration on various organ systems and on embryonic development. Even though the mammalian fetus develops in utero, while the 
externally fertilized zebrafish embryos develop ex utero, the embryos show striking similarities in the patterns of development, the genetic pathways that regulate the development and their responses to chemical. ${ }^{38,40}$ The gross development of the embryos appeared normal till day 1 in all the ILs (Fig. 2b-f). The surviving embryos appeared to have a normal body curvature, normal brain fold, a tubular and beating heart, normal otic vesicles, eyes, yolk and yolk extension and an age-appropriate number of somites. By day 3, normal embryos hatch out of their chorion and become free-swimming larvae. IL1 and IL2 treated larvae, most toxic for the cellular viability, appeared to have normal gross development till day 4 (Fig. $2 \mathrm{~h}$ and i), although the survival of IL1 and IL2 treated larvae, reduced by the later days. This may be attributed to general cell death. ILs have been implicated in disintegration of phospholipids bilayers, ${ }^{59}$ and causing oxidative damage in cells, which could accumulate over time, ultimately leading to lethality. ${ }^{60}$ However, it should noted that the toxicity observed in cultured cells do not correlate well with toxicity in whole organisms.
IL5 treated embryos hatched out normally but had slightly curved tails suggesting mild developmental defect (Fig. 2l). IL3 and IL4 exposed larvae were unable to hatch out efficiently (Fig. $2 \mathrm{j}$ and k). We manually dechorionated these embryos at day 2 and day 3 for closer observation and found a number of developmental abnormalities. The IL3 and IL4 treated embryos had a marked curvature of the body axis and pericardial (cellular sac enclosing the heart) edema (Fig. 2q and r). The IL3 and IL4 treated larvae did not have inflated swim bladders, which enable the animals to float and swim, when compared to the control larvae (Fig. 2t and u).

As evident in the control embryos (Fig. 2s), by day 4 the yolk (the nutrient store for the embryos till they start feeding) is mostly utilized and thins down. In contrast, the IL3 and IL4 treated embryos retained a large yolk indicative of poor nutrient absorption (Fig. 2t and u). By day 3, control larvae form jaw structures and begin actively feeding by day 5 . The IL3 and IL4 treated larvae had severely compromised jaw structures at day 4 (Fig. 2t, u, w and $\mathrm{x}$ ), which would preclude normal feeding.
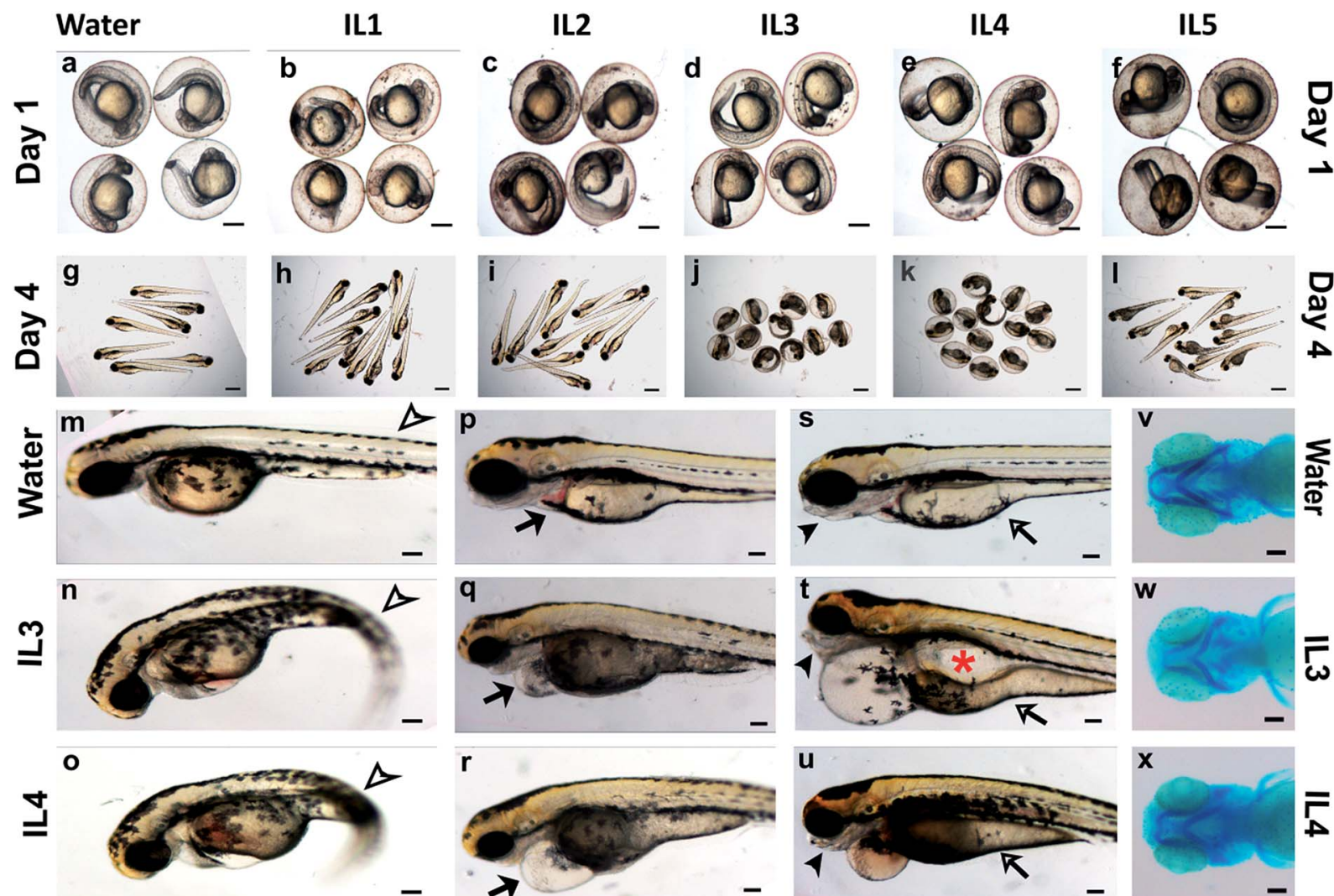

Day 2
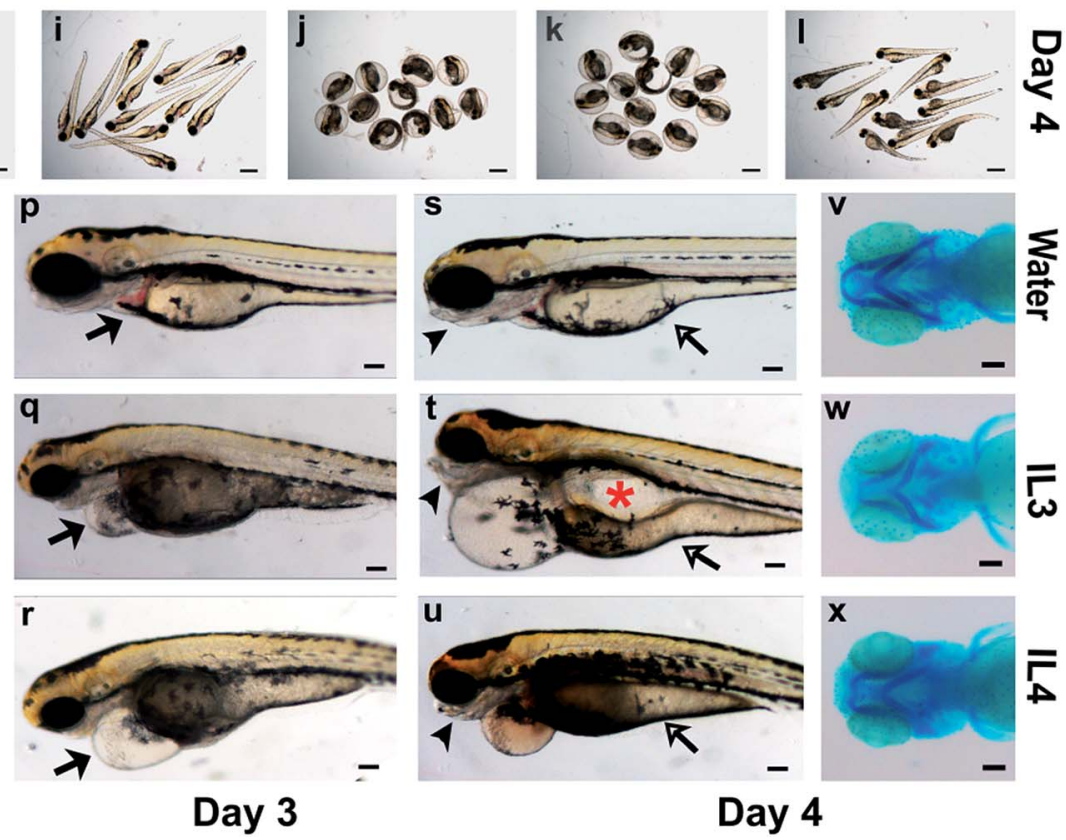

Day 4

Fig. 2 Teratogenicity assay of ionic liquids on zebrafish embryos. Embryos were exposed to sub-lethal concentrations of ILs from day 0 to day 4. (a-f) Embryos exposed to water, $2.5 \mathrm{mM}$ of IL1, IL2, IL3, IL4 and IL5 on day 1 are encased in the chorion and appear normal. (g-l) Embryos exposed to, IL1 (1.25 mM), IL2 (0.125 mM), IL3 (2.5 mM), IL4 (2.5 mM), IL5 (2.5 mM) on day 4. Embryos exposed to IL3 (j) and IL4 (k) fail to hatch out. Embryos exposed to $2.5 \mathrm{mM} \mathrm{IL3}$ and IL4 show a ventral curvature of the tail (black open arrowhead) compared to the control on day 2 ( $\mathrm{m}-0$ ). Embryos exposed to $2.5 \mathrm{mM} \mathrm{IL3}$ and IL4 show pericardial edema (black arrow) compared to control embryos on day 3 ( $p-r$ ). Embryos exposed to $2.5 \mathrm{mM}$ concentration of IL3 and IL4 show abnormal jaw (black arrowhead), swim bladder (red asterisk) and underutilized yolk (black open arrow) on day $4(\mathrm{~s}-\mathrm{u}$ ). Alcian blue stained cartilage of embryos exposed to water control, $2.5 \mathrm{mM}$ concentration of IL3 and IL4 on day 4 ( $\mathrm{v}-\mathrm{x}$ ). In each experiment, each condition or concentration the number $(N)$ of embryos used was $>50$. Each experiment was repeated on three or more independent occasions. Scale bars are $300 \mu \mathrm{m}(\mathrm{a}-\mathrm{f}), 400 \mu \mathrm{m}(\mathrm{g}-\mathrm{l})$ and $100 \mu \mathrm{m}(\mathrm{m}-\mathrm{x})$. 
These larvae also had smaller heads compared to the controls indicative of defects in brain development (Fig. 2p-r).

IL3 and IL4 elicited severe pericardial edema in embryos. To examine the effect of these ILs on the heart we exposed 1 day old embryos of the transgenic zebrafish line $T g$ (myl7:cerulean), to the ILs. The heart, in this transgenic animal, is marked with a green fluorescent protein and on day 2 can be seen to be fully functional with two chambers, atrium and ventricle, and the atrioventricular valve (Fig. 3b). Embryos treated with IL1, IL2 and IL5 had apparently normal hearts (Fig. 3c, d and g). The atrioventricular valve was not easily visualized in the IL3 and IL4 treated embryo hearts, which may be attributed to the mild stretching of the heart in the edematous pericardium that makes visualization of the valve difficult. However, the gross structure of the heart was normal in these embryos (Fig. 3e and f).

We determined the beat frequency of the hearts in these embryos at day 2. Zebrafish embryos are known to have a heart rate of approximately 120 beats per minute and the IL1 and IL2 treated embryos appeared normal in their beat frequency. However, we observed a statistically significant decrease in the heart rate of embryos treated with IL5 (2.5 mM concentration) (Fig. 3k), indicating compromised heart function. IL3 and IL4 treated embryos exhibited no periodic heartbeat with intermittent beats that were uncoordinated between the atrium and the ventricle. To dissect the effect on heart function independent of heart development, older embryos with a normally developed heart (2.5 day old embryos) were exposed to same concentration (2.5 mM) of IL3 and IL4 (Fig. 3i and j). After 16 hours of exposure, we observed similar characteristics in these embryos, with the beating frequency of zero beats per minute. This indicates these ILs specifically perturb the heart function.

Abnormal heart rate in treated embryos could be the result of impairment of contractile process by the ILs exposure. We determined the transcript levels of $n p p b$ (natriuretic peptide gene b), a cardiac hormone used as a classical hypertrophic marker. Its re-expression in cardiac stress condition is a clinical biomarker for diagnosis of heart failure and left-ventricular hypertrophy. ${ }^{61,62}$ IL3 and IL4 treated embryos had a significant upregulation of $n p p b$, indicative of cardiac stress ${ }^{63-65}$ (Fig. 31).
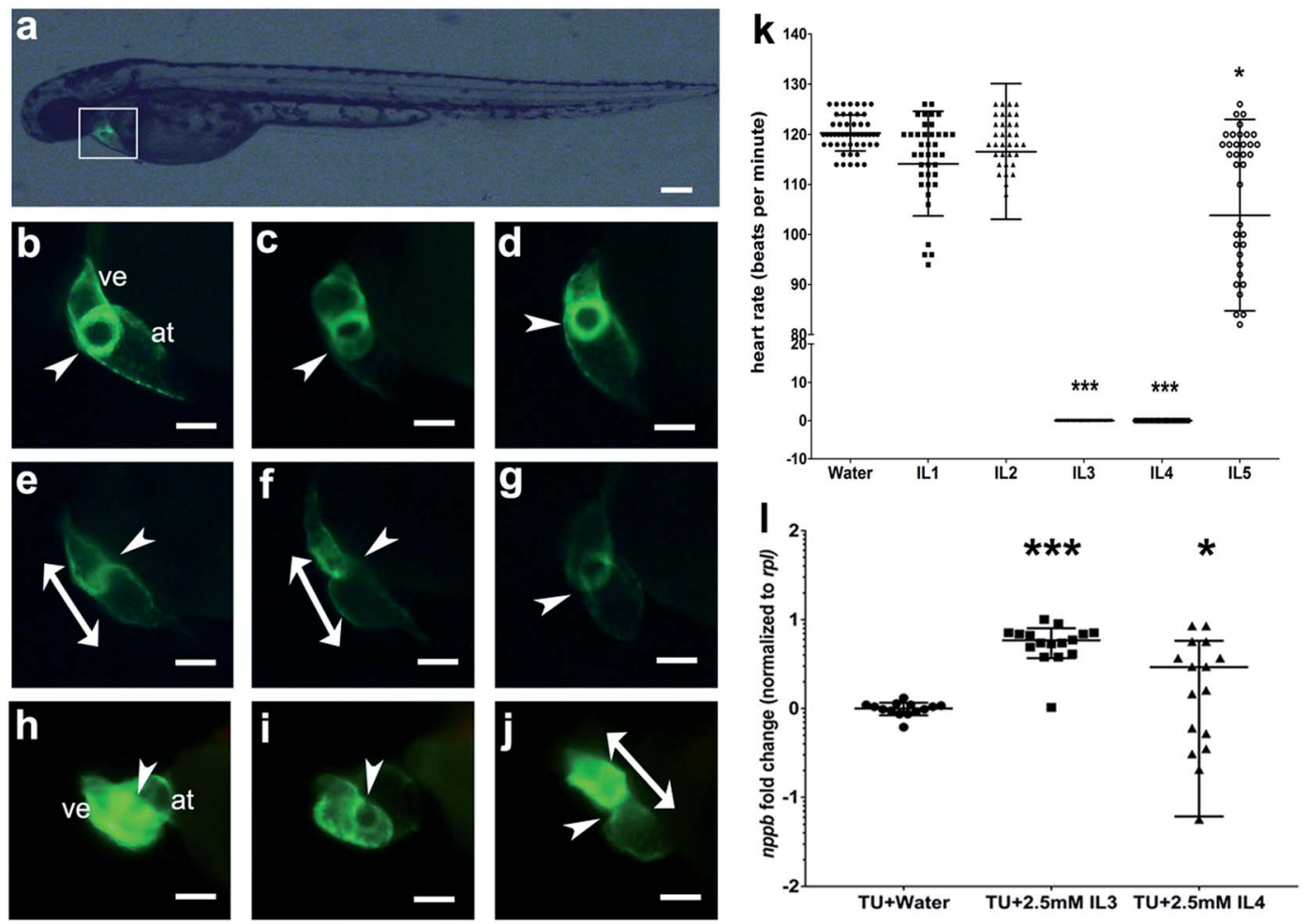

Fig. 3 Cardiotoxic effect of ionic liquids on zebrafish embryos. (a) Day 2 live embryo of $T g$ (myl7:cerulean) line marking the position of the fluorescent heart. (b-g) Magnified images of the heart as shown in the box (a) of embryos exposed to water (b), 1.25 mM IL1 (c), 0.125 mM IL2 (d) and $2.5 \mathrm{mM} \mathrm{IL5}(\mathrm{g})$ appear normal with atrium (at) and ventricle (ve) separated by the atrioventricular valve (white arrowhead). Embryos exposed to $2.5 \mathrm{mM} \mathrm{IL3}(\mathrm{e})$ and $2.5 \mathrm{mM} \mathrm{IL4}$ (f) show a stretching of the atrium-ventricle caused likely by the pericardial edema (white double arrow). (k) The heart rate (beats per minute) quantified for embryos exposed to water control and the ILs at the same concentration used for imaging. Data from 3 experiments, with $n=10$ in each experiment is plotted. ${ }^{*} p>0.0005$. (h-j) Magnified images of the heart day 3 live embryos of Tg(myl7:cerulean) line; embryos exposed to water (h), $2.5 \mathrm{mM} \mathrm{IL3} \mathrm{(i)} \mathrm{and} 2.5 \mathrm{mM} \mathrm{IL4} \mathrm{(j).} \mathrm{Embryos} \mathrm{exposed} \mathrm{to} 2.5 \mathrm{mM} \mathrm{IL3} \mathrm{(i)} \mathrm{and} 2.5 \mathrm{mM}$ IL4 (j) (treatment was given to 2.5 days old embryos, for 16 hours) show a stretching of the atrium-ventricle caused likely by the pericardial edema (white double arrow). (l) nppb transcript levels, upregulated in both IL3 and IL4 exposed embryos compared to the control. Transcript level fold change shown here in $Y$-axis, is logarithmic 2 scale. Data from 3 or more experiments, with $n=20$ or more in each experiment is plotted. * $p>0.05$. Graph pad prism was used for the analysis. For statistical significance of data, $p$-values were calculated by preforming unpaired student $t$-test for the data. Scale bars are $200 \mu \mathrm{m}(\mathrm{a})$ and $100 \mu \mathrm{m}(\mathrm{b}-\mathrm{j})$. 

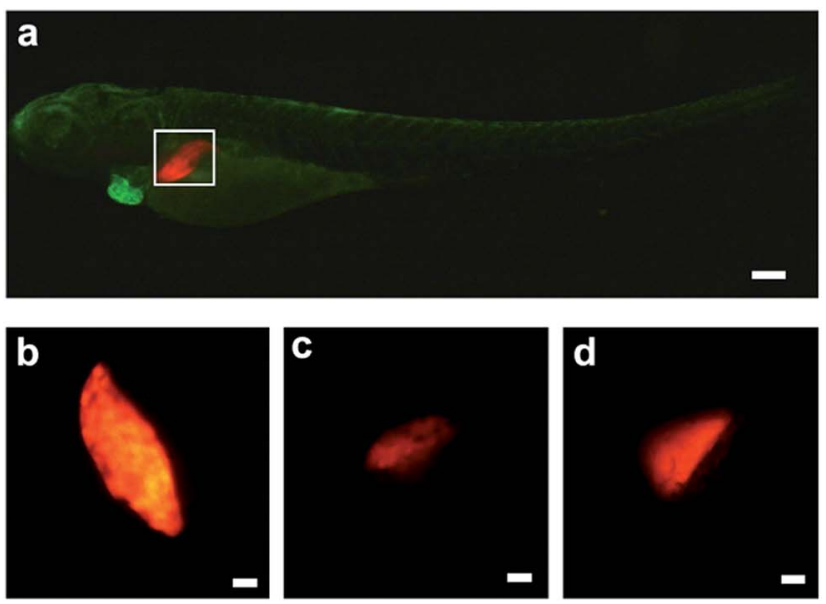

e

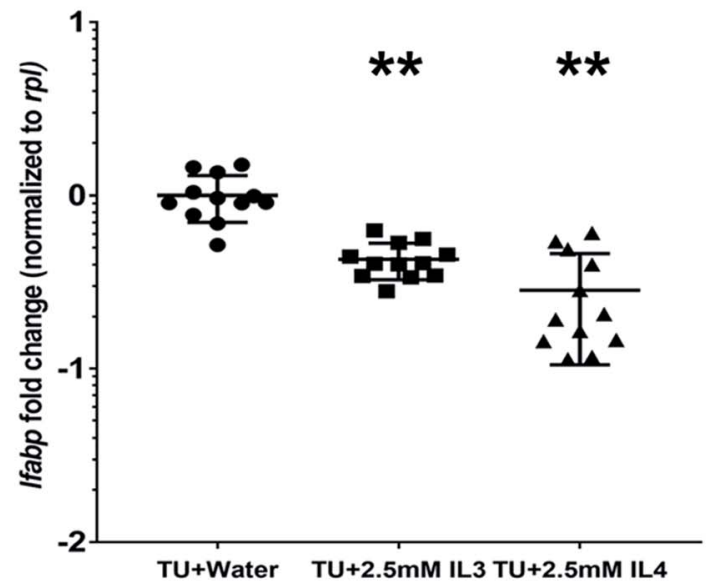

f

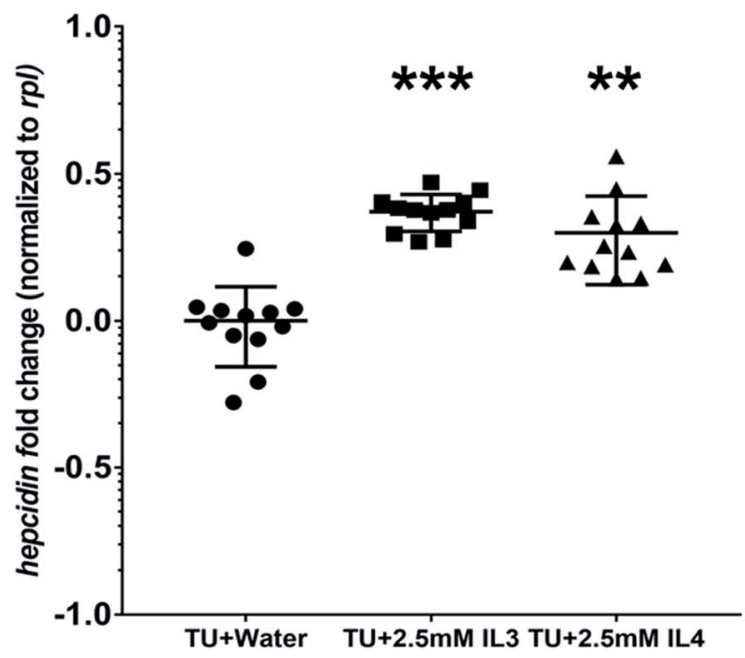

Fig. 4 Hepatotoxic effect of ionic liquids on zebrafish embryos. (a) Day 3 live embryo of Tg(fabp10a:GAL4-VP16)::(UAS:nfsB-Cherry) line marking the position of the fluorescent liver. $(b-d)$ Magnified images of the liver as shown in the box (a) of embryos exposed to water (b), $2.5 \mathrm{mM} \mathrm{IL3}$ (c) and $2.5 \mathrm{mM} \mathrm{IL4}$ (d). Embryos exposed to $2.5 \mathrm{mM} \mathrm{IL3} \mathrm{(c)}$ and $2.5 \mathrm{mM} \mathrm{IL4}$ (d) (treatment was given to 3 day old embryos, for 16 hours) show reduced fluorescent intensity and liver size compared to water control embryo (b). (e) fabp10, transcript levels are severely decreased in these IL exposed embryos. (f) Hepcidin transcript levels are severely increased in these IL exposed embryos. Transcript level fold change shown here in $Y$-axis, is logarithmic 2 scale. Data from 3
Liver is the most important metabolic center of the body and damage to the liver can have life-threatening consequences. Liver is also highly responsive to toxic stress from drugs and toxins. We used a double transgenic animal, $\mathrm{Tg}$ (fabp10a:GAL4VP16)::(UAS:nfsB-Cherry) which selectively marks the liver hepatocytes with a red fluorescent protein to assess the effect of the ILs on the liver. We exposed the larvae of this transgenic line at day 3 (to bypass early developmental effects) to IL3 and IL4 and observed a significant reduction in liver size in the treated larvae, indicating hepatocellular death (Fig. 4c and d). Quantitative real-time PCR confirmed a decrease in the transcript level of fabp10, a marker for hepatocytes, ${ }^{66}$ in IL3 and IL4 treated larvae (Fig. 4e). We further assayed the transcript levels of hepcidin, a liver hormone that is known to be induced in response to liver stress ${ }^{67}$ and found a 1.4 fold induction in the IL3 and IL4 treated larvae (Fig. 4f).

\section{Conclusion}

In this study, a small set of ionic liquids was assessed for their cellular and whole organism toxicity. There are large numbers of studies which have analyzed the cytotoxic effect of ILs. Majority of them have focused on ILs containing imidazoliumbased cationic head group. The common features contributing to cytotoxicity were different alkyl chain lengths in the cationic core and the various combination of anions. Other than these, molecular structural factors such as aromaticity of the cation also contribute to cytotoxicity; ILs containing imidazolium and pyridinium groups as cations are, in general, more toxic than the non-aromatic ILs. This is well supported by our data. Aromatic cationic core-containing IL1, IL2, IL4 and IL5 were also more developmentally toxic than non-aromatic cationic core-containing IL3. Symmetry of the IL as well as charge distribution in the cation and heavy atom count in the anion also add to the cytotoxicity. As symmetry of the cation decreases, the cytotoxicity was predicted to increase. ${ }^{24}$ In terms of symmetry, a pyridinium cation has a higher symmetry than an imidazolium cation and hence, imidazolium-based ILs are comparatively more toxic can pyridinium based ILs. ${ }^{24}$ In our data, IL5 containing a pyridinium group is less toxic than IL1 IL2 and IL-4, all containing imidazolium group as their cationic cores.

IL3 and IL4 were the most teratogenic ILs among the ones tested in our study causing a similar functional breakdown of the heart and toxicity in the liver. IL3 and IL4 have the same anion but very different cationic core. While IL2 and IL4 sharing the same cationic component, showed no similarity in their teratogenic potential, suggesting that the anionic component is a critical determinant of their biological activity. On the other hand, IL1 and IL5 that share the same anionic component, elicited different effects on the heart and general development.

experiments, with $n>10$ in each experiment is plotted. ${ }^{*} p>0.05$. Graph pad prism was used for the analysis. For statistical significance of data, $p$-values were calculated by preforming unpaired student $t$-test for the data. Scale bars are $200 \mu \mathrm{m}$ (a) and $50 \mu \mathrm{m}(\mathrm{b}-\mathrm{d})$. 
These observations suggest that both the anion and the cation may be important in interacting with biological systems in different molecular contexts. The degree of toxicity varies according to dose and exposure period of ILs. Lethal and nonlethal effects on the zebrafish embryos also could be because of ability of ILs to be absorbed and/or metabolized in the fish.

Although ionic liquids are considered a 'greener' alternative to traditional organic solvents, their effect on the environment and specifically on human health is still not well understood. Our study should serve as a preliminary step in probing the effects of this group of molecules on biological systems, especially using vertebrate models.

\section{Author contributions}

The manuscript was written through contributions of all authors. CS \& SM conceived the work and conceptualized the experimental plan. AP did the biological assays on zebrafish. AP and SR were involved in the zebrafish maintenance and breeding for experiments. MKE performed all the cell culture experiments. All authors have given approval to the final version of the manuscript.

\section{Funding sources}

Council of Scientific and Industrial Research (CSIR), New Delhi is acknowledged for the financial support through NCLB-IGIB Joint Research Initiative (BSC0124).

\section{Acknowledgements}

The authors acknowledge Michael Nonet for providing the $p C H$ Gtwy G4VP16 plasmid (http://www.dbbs.wustl.edu/RIB/NonetMicL) and Marnie E. Halpern for providing UAS: $n f s B-m C h e r r y{ }^{50}$ construct.

\section{References}

1 P. Walden, Bull. Acad. Imp. Sci. St.-Petersbourg, 1914, 8, 405422.

2 H. Zhao, Chem. Eng. Commun., 2006, 193, 1660-1677.

3 S. Cantone, U. Hanefeld and A. Basso, Green Chem., 2007, 9, 954-971.

4 A. P. M. Tavares, O. Rodriguez and E. A. Macedo, Biotechnol. Bioeng., 2008, 101, 201-207.

5 P. Domínguez de María, Angew. Chem., Int. Ed., 2008, 47, 6960-6968.

6 P. Lozano, T. De Diego and J. L. Iborra, Chim. Oggi, 2007, 25, 76.

7 N. V. Plechkova and K. R. Seddon, Chem. Soc. Rev., 2008, 37, 123-150.

8 P. Wasserscheid and T. Welton, Ionic liquids in synthesis, Wiley Online Library, 2008.

9 K. S. Egorova, E. G. Gordeev and V. P. Ananikov, Chem. Rev., 2017, DOI: 10.1021/acs.chemrev.6b00562.

10 K. M. Docherty and C. F. Kulpa Jr, Green Chem., 2005, 7, 185-189.

11 J. N. Pendleton and B. F. Gilmore, Int. J. Antimicrob. Agents, 2015, 46, 131-139.
12 V. Kumar and S. V. Malhotra, Bioorg. Med. Chem. Lett., 2009, 19, 4643-4646.

13 S. V. Malhotra and V. Kumar, Bioorg. Med. Chem. Lett., 2010, 20, 581-585.

14 H. L. Chen, H. F. Kao, J. Y. Wang and G. T. Wei, J. Chin. Chem. Soc., 2014, 61, 763-769.

15 D. J. Couling, R. J. Bernot, K. M. Docherty, J. K. Dixon and E. J. Maginn, Green Chem., 2006, 8, 82-90.

16 J. Arning, S. Stolte, A. Böschen, F. Stock, W.-R. Pitner, U. Welz-Biermann, B. Jastorff and J. Ranke, Green Chem., 2008, 10, 47-58.

17 M. T. Garcia, N. Gathergood and P. J. Scammells, Green Chem., 2005, 7, 9-14.

18 A. S. Wells and V. T. Coombe, Org. Process Res. Dev., 2006, 10, 794-798.

19 K. J. Kulacki and G. A. Lamberti, Green Chem., 2008, 10, 104-110. 20 K. A. Kurnia, T. E. Sintra, C. M. Neves, K. Shimizu, J. N. C. Lopes, F. Gonçalves, S. P. Ventura, M. G. Freire, L. M. Santos and J. A. Coutinho, Phys. Chem. Chem. Phys., 2014, 16, 19952-19963.

21 C. Wang, Z. Wei, L. Wang, P. Sun and Z. Wang, Ecotoxicol. Environ. Saf., 2015, 115, 112-118.

22 S. Stolte, J. Arning, U. Bottin-Weber, M. Matzke, F. Stock, K. Thiele, M. Uerdingen, U. Welz-Biermann, B. Jastorff and J. Ranke, Green Chem., 2006, 8, 621-629.

23 J. Ranke, K. Mölter, F. Stock, U. Bottin-Weber, J. Poczobutt, J. Hoffmann, B. Ondruschka, J. Filser and B. Jastorff, Ecotoxicol. Environ. Saf., 2004, 58, 396-404.

24 M. H. Fatemi and P. Izadiyan, Chemosphere, 2011, 84, 553563.

25 J. Ranke, A. Müller, U. Bottin-Weber, F. Stock, S. Stolte, J. Arning, R. Störmann and B. Jastorff, Ecotoxicol. Environ. Saf., 2007, 67, 430-438.

26 S. P. Ventura, C. S. Marques, A. A. Rosatella, C. A. Afonso, F. Gonçalves and J. A. Coutinho, Ecotoxicol. Environ. Saf., 2012, 76, 162-168.

27 B. Yoo, J. K. Shah, Y. Zhu and E. J. Maginn, Soft Matter, 2014, 10, 8641-8651.

28 T. W. Schultz, M. T. D. Cronin and T. I. Netzeva, J. Mol. Struct.: THEOCHEM, 2003, 622, 23-38.

29 M. Alvarez-Guerra and A. Irabien, Green Chem., 2011, 13, 1507-1516.

30 X. Wang, C. A. Ohlin, Q. Lu, Z. Fei, J. Hu and P. J. Dyson, Green Chem., 2007, 9, 1191-1197.

31 A. Santos, B. Ribeiro, D. Alviano and M. Coelho, RSC Adv., 2014, 4, 37157-37163.

32 R. F. Frade and C. A. Afonso, Hum. Exp. Toxicol., 2010, 29, 1038-1054.

33 J. Jodynis-Liebert, M. Nowicki, M. Murias, T. Adamska, M. Ewertowska, M. Kujawska, H. Piotrowska, A. Konwerska, D. Ostalska-Nowicka and J. Pernak, Regul. Toxicol. Pharmacol., 2010, 57, 266-273.

34 G. Dumitrescu, L. P. Ciochină, L. Stana, I. Cre $\square$ escu, R. Popescu, N. M. Filimon and O. S. Voia, Rom. Biotechnol. Lett., 2014, 19, 8925-8934.

35 J. Pernak, A. Czepukowicz and R. Pozniak, Ind. Eng. Chem. Res., 2001, 40, 2379-2383. 
36 M. M. Bailey, M. B. Townsend, P. L. Jernigan, J. Sturdivant, W. L. Hough-Troutman, J. F. Rasco, R. P. Swatloski, R. D. Rogers and R. D. Hood, Green Chem., 2008, 10, 1213-1217. 37 X.-Y. Li, J. Zhou, M. Yu, J.-J. Wang and Y. C. Pei, Ecotoxicol. Environ. Saf., 2009, 72, 552-556.

38 C. A. MacRae and R. T. Peterson, Nat. Rev. Drug Discovery, 2015, 14, 721-731.

39 S. Basu and C. Sachidanandan, Chem. Rev., 2013, 113, 79527980.

40 G. J. Lieschke and P. D. Currie, Nat. Rev. Genet., 2007, 8, 353367.

41 Y. J. Dai, Y. F. Jia, N. Chen, W. P. Bian, Q. K. Li, Y. B. Ma, Y. L. Chen and D. S. Pei, Environ. Toxicol. Chem., 2014, 33, 11-17.

42 C. Pretti, C. Chiappe, D. Pieraccini, M. Gregori, F. Abramo, G. Monni and L. Intorre, Green Chem., 2006, 8, 238-240.

43 S.-K. Ruokonen, C. Sanwald, M. Sundvik, S. Polnick, K. Vyavaharkar, F. Duša, A. J. Holding, A. W. T. King, I. Kilpeläinen, M. Lämmerhofer, P. Panula and S. K. Wiedmer, Environ. Sci. Technol., 2016, 50, 7116-7125.

44 Z. Du, L. Zhu, M. Dong, J. Wang, J. Wang, H. Xie, T. Liu and Y. Guo, Arch. Environ. Contam. Toxicol., 2014, 67, 261-269.

45 C. Pretti, C. Chiappe, I. Baldetti, S. Brunini, G. Monni and L. Intorre, Ecotoxicol. Environ. Saf., 2009, 72, 1170-1176.

46 M. El-Harbawi, Procedia Chem., 2014, 9, 40-52.

47 T. Mosmann, J. Immunol. Methods, 1983, 65, 55-63.

48 M. Westerfield, The zebrafish book: a guide for the laboratory use of zebrafish (Danio rerio), University of Oregon Press, 2000.

49 C. B. Kimmel, W. W. Ballard, S. R. Kimmel, B. Ullmann and T. F. Schilling, Dev. Dyn., 1995, 203, 253-310.

50 J. M. Davison, C. M. Akitake, M. G. Goll, J. M. Rhee, N. Gosse, H. Baier, M. E. Halpern, S. D. Leach and M. J. Parsons, Dev. Biol., 2007, 304, 811-824.
51 M. W. Pfaffl, Nucleic Acids Res., 2001, 29, e45.

52 K. J. Livak and T. D. Schmittgen, Methods, 2001, 25, 402-408. 53 G. Dingerkus and L. D. Uhler, Stain Technol., 1977, 52, 229232.

54 A. L. Monteiro, F. K. Zinn, R. F. de Souza and J. Dupont, Tetrahedron: Asymmetry, 1997, 8, 177-179.

55 D. H. Cheng, X. W. Chen, J. H. Wang and Z. L. Fang, Chem.Eur. J., 2007, 13, 4833-4839.

56 L. S. Wang, L. Wang, L. Wang, G. Wang, Z. H. Li and J. J. Wang, Environ. Toxicol., 2009, 24, 296-303.

57 S. P. Ventura, A. M. Goncalves, T. Sintra, J. L. Pereira, F. Goncalves and J. A. Coutinho, Ecotoxicology, 2013, 22, 112.

58 P. Stepnowski, A. Skladanowski, A. Ludwiczak and E. Laczyńska, Hum. Exp. Toxicol., 2004, 23, 513-517.

59 K. O. Evans, Colloids Surf., A, 2006, 274, 11-17.

60 M. Cvjetko, K. Radosevic, A. Tomica, I. Slivac, J. VorkapicFurac and V. G. Srcek, Arh. Hig. Rada Toksikol., 2012, 63, 15-20.

61 D. L. Dries, Heart Fail Clin, 2010, 6, 55-64.

62 S. Hayek and M. Nemer, Cardiovasc. Ther., 2011, 29, 362-376. 63 D. G. Gardner, Trends Endocrinol. Metab., 2003, 14, 411-416.

64 E. N. Olson and M. D. Schneider, Genes Dev., 2003, 17, 19371956.

65 J. R. Becker, T. Y. Robinson, C. Sachidanandan, A. E. Kelly, S. Coy, R. T. Peterson and C. A. MacRae, Cardiovasc. Res., 2012, 93, 463-470.

66 Y. Zhang, K. Liu, H. M. Hassan, H. Guo, P. Ding, L. Han, Q. He, W. Chen, C.-D. Hsiao and L. Zhang, Antimicrob. Agents Chemother., 2016, 01693-01616.

67 P. An, H. Wang, Q. Wu, X. Guo, A. Wu, Z. Zhang, D. Zhang, X. Xu, Q. Mao, X. Shen, L. Zhang, Z. Xiong, L. He, Y. Liu, J. Min, D. Zhou and F. Wang, Sci. Rep., 2015, 5, 13106. 